\title{
A Method of Model Matching Control for Continuous-Time Systems in the Presence of Arbitrarily Bounded Disturbances
}

\author{
Member Jianming LU (Chiba University) \\ Non-member Kyohei ISHIHATA (Chiba University) \\ Non-member Takashi YAHAGI (Chiba University)
}

A method of model matching control for continuous-time systems in the presence of arbitrarily bounded disturbances is proposed. The proposed control system consists of two feedback loops: one performs the model-matching control and the other includes an error feedback controller which could be fully utilized to reduce the effect of external disturbances. In this paper, an explicit design procedure for achieving perfect model matching control with minimal sensitivity will be proposed. Furthermore, we can assure that the system input and output remain bounded at any time for continuous-time system in the presence of arbitrarily bounded disturbances. Finally, the results of computer simulation are presented to illustrate the effectiveness of the proposed method.

Keywords: model matching controller, disturbance, continuous-time system, minimal sensitivity

\section{Introduction}

The ultimate goal of the model matching is to match the transfer function of the closed loop system to some desired transfer function ${ }^{(1)}$. Due to the fact that the desired system model is usually characterized in terms of a transfer function, it is natural to treat the design of perfect model matching control in the frequency domain directly rather than in the time domain. Thus, recent work concerning perfect model matching control is mostly done in the frequency domain ${ }^{(2) \sim(4)}$. Furthermore, it is now well known that $H_{\infty}$-control problem including both sensitivity minimization and robust stabilization is reduced to a mathematical problem called the model matching problem in $H_{\infty}{ }^{(5)}$. Interpolation approach and approximation approach has been proposed to solve the model-matching problem. Both approaches are mathematically equivalent but they are different in computational aspect. The interpolation approach is conceptually appealing ${ }^{(6),(7)}$ but unsuccessful in providing a powerful computational algorithm. The approximation method has made remarkable progress from computational point of view ${ }^{(8) \sim(10)}$. An extension of the interpolation technique called directional interpolation is a variation of the Nevanlinna-Pick problem $^{(11),(12)}$. This method can be used to design minimal-order controller in the frequency domain ${ }^{(6)}$. State-space version of the classical interpolation theory has been developed for the simplest model-matching problem.

In this paper we introduce a method to solve the perfect model matching problem for continuous-time systems in the presence of disturbances based upon the pole-zero placement method. In this method, we introduce the output loop compensator for the compen- sation of external disturbances. Furthermore, in this paper, an explicit design procedure for achieving perfect model matching control with minimal sensitivity will be proposed. Here, we adopt the polynomial approach for designing the controller ${ }^{(13) \sim(16)}$. Section 2 states the problem. In section 3 , we precisely state the problem of minimal sensitivity perfect model matching control and give a complete solution and an explicit design procedure. The results of computer simulation are presented in Section 4.

\section{Problem Statement}

Let the single-input single-output continuous-time plant be given by

$$
y(t)=G(s) u(t)+w(t)
$$

where

$$
\begin{aligned}
& G(s)=\frac{B(s)}{A(s)} \cdots \cdots \cdots \\
& A(s)=s^{n}+\sum_{i=1}^{n-1} a_{n-i} s^{n-i} \\
& B(s)=\sum_{i=0}^{m} b_{m-i} s^{m-i}
\end{aligned}
$$

$A(s)$ and $B(s)$ are stable coprime polynomials in $s$ and $m \leq n . u(t)$ and $y(t)$ are the plant input and output, respectively. $w(t)$ is the external arbitrarily bounded disturbances. Moreover, the continuous-time plant is a minimum phase system ( $B(s)$ is a stable polynomial ).

The reference model is described by

$$
y_{m}(t)=\frac{B_{m}(s)}{A_{m}(s)} r(t)
$$


where

$$
\begin{aligned}
& A_{m}(s)=s^{\nu}+\sum_{i=1}^{\nu-1} a_{m(\nu-i)} s^{\nu-i} \\
& B_{m}(s)=\sum_{i=0}^{\mu} b_{m(\mu-i)} s^{\mu-i}
\end{aligned}
$$

$A_{m}(s)$ is a stable polynomial and $B_{m}(s)$ is a polynomial, and $\nu \geq \mu$ and $\nu-\mu \geq n-m$ are satisfied. $r(t)$ is the reference input and $y_{m}(t)$ is the reference model output. Furthermore, the polynomial $A_{m}(s)$ and $B_{m}(s)$ are relatively coprime polynomials. The objective of the control is to design a controller such that the transfer function of the closed-loop from the reference input $r(t)$ to the plant output $y(t)$ becomes $B_{m}(s) / A_{m}(s)$ and the system should be robustly stable. Thus, in steady state, output $y(t)$ of the plant will converge to the reference model output $y_{m}(t)$

\section{Controller Design}

Now, using the pole-zero placement method, it is possible to design a controller such that the closed-loop transfer function of the system from the reference input $r(t)$ to plant output $y(t)$ matches some desired transfer function. If $T_{0}(s)$ is an asymptotically stable polynomial, then there exist unique polynomials $R(s)$ and $S(s)$, which satisfy the following Diophantine equation ${ }^{(13),(17)}$

$$
A(s) R(s)+B(s) S(s)=A_{m}(s) T_{0}(s)
$$

where, $\operatorname{deg} T_{0}(s)=l(l>n-1), \operatorname{deg} R(s)=\nu+l-n$ and $\operatorname{deg} S(s)=n-1 . T_{0}(s)$ is the part of the desired closed-loop characteristic polynomial which should not influence the reference tracking. It is interpreted as observer polynomial. The polynomial $A_{m}(s)$ is the desired closed-loop characteristic polynomial. Here, it is the denominator polynomial of the reference model.

We define the control input $u(t)$ by

$$
u(t)=T_{2}(s)\left[r(t)-\epsilon^{0}(t)\right]-T_{1}(s) y(t) \cdots \cdots
$$

where

$$
\begin{aligned}
& T_{1}(s)=\frac{S(s)}{R(s)} \cdots \ldots \\
& T_{2}(s)=\frac{T_{0}(s) B_{m}(s)}{B(s) R(s)} \\
& e^{0}(t)=\frac{N(s)}{M(s)} e(t) \ldots \\
& e(t)=y(t)-y_{m}(t) \ldots
\end{aligned}
$$

and $M(s)$ and $N(s)$ are polynomials. This perfect model matching controller in eq. (5) could be thought of as a combination of feedback having the transfer function $T_{1}(s)$, a feedforward with the transfer function $T_{2}(s)$ and the output loop compensator $N(s) / M(s)$.

The block diagram of the proposed system is shown in Fig. 1.

From eqs. (1), (4), the following equation holds.

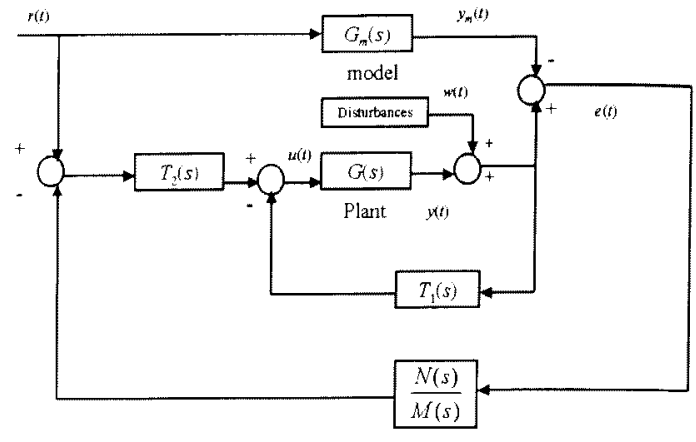

Fig. 1. Block diagram of the proposed system

$$
\begin{aligned}
y(t)= & \frac{B(s) R(s)}{T_{0}(s) A_{m}(s)} u(t)+\frac{B(s) S(s)}{T_{0}(s) A_{m}(s)} y(t) \\
& +\frac{A(s) R(s)}{T_{0}(s) A_{m}(s)} w(t) \ldots \ldots \ldots \ldots \ldots
\end{aligned}
$$

Using eqs. (5) (9), it is possible to write eq.(10) as

$$
\begin{aligned}
y(t)= & \frac{1}{T_{0}(s) A_{m}(s)}\left[T_{0}(s) B_{m}(s) r(t)\right. \\
& \quad-T_{0}(s) B_{m}(s) \frac{N(s)}{M(s)} y(t) \\
+ & \left.T_{0}(s) B_{m}(s) \frac{N(s)}{M(s)} y_{m}(t)+A(s) R(s) w(t)\right]
\end{aligned}
$$

Moreover, if the polynamial $\left[A_{m}(s) M(s)+B_{m}(s) N(s)\right]$ and $M(s)$ are stable, by using eq. (11), the following relation can be derived

$$
\begin{aligned}
& y(t)=\frac{B_{m}(s)}{A_{m}(s)} r(t) \\
& +\frac{M(s) R(s) A(s)}{T_{0}(s)\left(A_{m}(s) M(s)+B_{m}(s) N(s)\right)} w(t) \cdots
\end{aligned}
$$

From eq. (12), the sensitivity function relating the disturbance $w(t)$ to output $y(t)$ can be given by

$$
S S(s)=\frac{M(s) R(s) A(s)}{T_{0}(s)\left(A_{m}(s) M(s)+B_{m}(s) N(s)\right)}
$$

which can be interpreted as a sensitivity function relating output to disturbance. To have perfect model matching control with minimal sensitivity to disturbance $w(t)$, it is required to find the design parameters $M(s)$ and $N(s)$ which not only satisfy the stability of $A_{m}(s) M(s)+B_{m}(s) N(s)$, but also minimize the following meaningful measure of sensitivity in the low frequency region.

$$
\begin{aligned}
J= & \left\|\frac{M(s) R(s) A(s) V(s)}{T_{0}(s)\left(A_{m}(s) M(s)+B_{m}(s) N(s)\right)}\right\|_{\infty} \\
& s=j \omega, \quad \forall \omega \ldots \ldots \ldots \ldots \ldots \ldots \ldots
\end{aligned}
$$

where $W(s)$ is the Laplace transform of $w(t), \quad$ | $W(j \omega)|\leq| V(j \omega) \mid$, and $\|\cdot\|_{\infty}$ is defined as

$$
\|G(s)\|_{\infty}=\sup _{\omega}|G(j \omega)|
$$


For a given any stable polynomial $T_{3}(s), \tilde{M}(s)$ nd $\tilde{N}(s)$ always exist, which satisfy the following quation ${ }^{(18)}$

$$
T_{3}(s)=A_{m}(s) \tilde{M}(s)+B_{m}(s) \tilde{N}(s)
$$

there deg $T_{3}(s)=\xi$. The degrees of the polynomials $\tilde{A}(s)$ and $\tilde{N}(s)$ can be fixed as $\operatorname{deg} \tilde{M}(s)=\xi-\nu$ and ieg $\tilde{N}(s)=\nu-1$, respectively.

Furthermore, we define two polynomials $M(s)$ and $\mathrm{V}(s)$ as

$$
\begin{aligned}
M(s) & =\tilde{M}(s)-L(s) B_{m}(s) \\
N(s) & =\tilde{N}(s)+L(s) A_{m}(s)
\end{aligned}
$$

In this paper, we introduce a new idea to design the jarameters of the controller. The parameters of the conroller are designed using polynomials $\tilde{M}(s)$ and $\tilde{N}(s)$, btained from the Diophantine equation (16). We can btain these polynomials such that the control input sbtained from eq. (5) robustly stabilizes the plant and Ilso the closed-loop transfer function from the reference nput $r(t)$ to output $y(t)$ matches some desired transfer unction, as in eq. (3).

Using eqs. $(13),(16) \sim(18)$ the sensitivity function :elating output $y(t)$ to the disturbance $w(t)$ can be given sy

$$
S S(s)=\frac{\left(\tilde{M}(s)-L(s) B_{m}(s)\right) R(s) A(s)}{T_{0}(s) T_{3}(s)} \cdots
$$

The effect of $w(t)$ can be minimized by choosing a suitable sensitivity transfer function $\|S(s) V(s)\|_{\infty}$. Here, we define the following criterion

$$
\begin{aligned}
J & =\left\|\frac{\left(\tilde{M}(s)-L(s) B_{m}(s)\right) R(s) A(s) V(s)}{T_{0}(s) T_{3}(s)}\right\|_{\infty} \\
& =\|S S(s) V(s)\|_{\infty}<\gamma, \quad s=j \omega, \quad \forall \omega \cdots
\end{aligned}
$$

where $\gamma$ is a small constant $\gamma>0$.

Then, the problem becomes equivalent to finding the ational function $L(s)$ such that $J$ given in eq. (20) can Je satisfy $J<\gamma$. In this way, the rational function $L(s)$ $s$ independent of the disturbance and the plant, and the nodel matching will not be destroyed. Furthermore, the rational function $L(s)$ can easily be implemented sy using following methods.

3.1 In case of stable polynomial $A(s)$ When $A(s)$ is a stable polynomial, the transfer function $L(s)$ san be obtained from the following relation

$$
L(s)=\frac{\tilde{M}(s) R(s) A(s) V_{n}(s)-\rho T_{0}(s) T_{3}(s) V_{d}(s)}{B_{m}(s) R(s) A(s) V_{n}(s)}
$$

where $V(s)=V_{n}(s) / V_{d}(s)$. $\rho$ is an arbitrarily chosen zonstant and $\rho<\gamma$. Now, using eqs. (20) and (21), the ninimal value of $J$ can be given by

$$
J=|\rho|<\gamma
$$

Jere $J$ depends upon $\rho$ and $\rho$ can be selected arbitrarily. Thus $J$ can be minimized upto the desired level.

Furthermore, substituting eq. (21) into eqs. 18 ), it can be shown that $N(s) / M(s)$ is stable.
3.2 In case of unstable polynomial $A(s)$ In the case when $A(s)$ is an unstable polynomial, $A(s)$ is divided into a stable polynomial component $A^{+}(s)$ and unstable polynomial component $A^{-}(s)$, where $A(s)=$ $A^{+}(s) A^{-}(s)$. The degree of $A^{+}(s)$ is $n_{1}$ and the degree of $A^{-}(s)$ is $n_{2}$, and $n_{1}+n_{2}=n$. Since $A^{-}(-s)$ is a stable polynomial, the transfer function $L(s)$ can be chosen as

$$
\begin{aligned}
L(s)= & \frac{\tilde{M}(s) R(s) A^{+}(s) A^{-}(-s) V_{n}(s)}{B_{m}(s) R(s) A^{+}(s) A^{-}(-s) V_{n}(s)} \\
& -\frac{\rho T_{0}(s) T_{3}(s) V_{d}(s)}{B_{m}(s) R(s) A^{+}(s) A^{-}(-s) V_{n}(s)}
\end{aligned}
$$

Now, using eq. (20) and eq. (23), the minimal value of $J$ can be given by

$$
J=\left\|\rho \frac{A^{-}(s)}{A^{-}(-s)}\right\|_{\infty}=|\rho|<\gamma
$$

and $J$ can be minimized upto the desired level.

Furthermore, substituting eq. (23) into eqs. (17), (18), it can be shown that $N(s) / M(s)$ is stable.

Consequently, a simple and direct design algorithm for achieving perfect model matching control with minimal sensitivity can be outlined as follows.

\section{Design Algorithm:}

Step 1: Choose a stable polynomials $T_{0}(s), T_{3}(s)$.

Step 2: Solve Diophantine equations (4), (16).

Step 3: Choose $V(s)$ and the small constant $p$.

Step 4: Obtain $L(s)$ from eq. (21) or eq. (23).

Step 5: Obtain $M(s)$ and $N(s)$ from eqs. (17), (18).

Step 6: Then the controller takes the from eq. (5).

\section{Simulation Results}

In this section, the results of simulations are presented to give an indication of the performance of the model matching scheme.

Example 1: Let us consider the case when the polynomial $A(s)$ has stable poles, and the plant with external disturbance described by the following equation

$$
y(t)=\frac{B(s)}{A(s)} r(t)+w(t)=\frac{s^{2}+7 s+6}{s^{2}+5 s+6} r(t)+w(t)
$$

where $A(s)$ has two stable poles, at -2 and -3 , and $B(s)$ has two stable zeros at -1 and -6 . Furthermore, the disturbance is $w(t)=0.5, t>0$.

The reference model $G_{m}(s)$ is given by

$$
G_{m}(s)=\frac{s^{2}+12 s+35}{s^{2}+12 s+32}
$$

Choose stable polynomials $T_{0}(s)=s^{2}+4 s+3$, $T_{3}(s)=s^{3}+6 s^{2}+11 s+6$.

Furthermore, choose $V(s)=2.011(s+1) /(s+0.1005)$, the small constant $\rho=0.01$.

Form eq. (21), we obtain

$$
L(s)=\frac{-16 s^{2}-184 s-504}{s^{3}+19 s^{2}+119 s+245}
$$




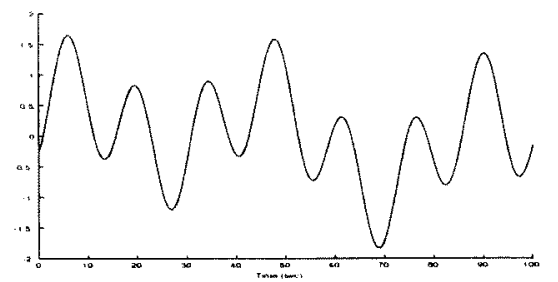

(a) $--: y(t)$ (plant output); 一: $y_{m}(t)$ (reference output)

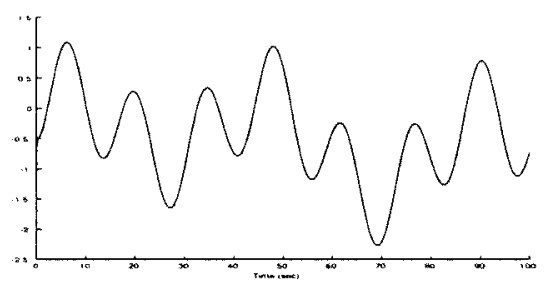

(b) $u(t)$ (plant input)

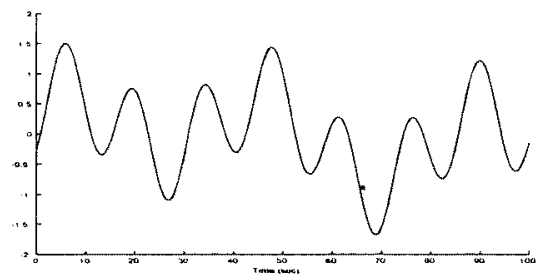

(c) $r(t)$ (reference input)

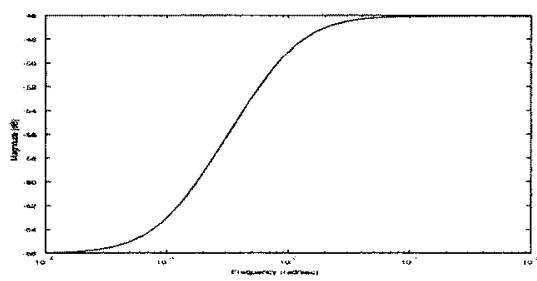

(d) $S S(s)$ (sensitivity function)

Fig. 2. Result of model matching control with step disturbance $(w(t)=0.5, \rho=0.01)$

Solve Diophantine equation (16), and using eqs. (17), (18), then $N(s) / M(s)$ is

$$
\begin{aligned}
& \frac{N(s)}{M(s)}=\frac{200.1 s^{5}+4799 s^{4}+4.237 \times 10^{4} s^{3}}{s^{5}+26.1 s^{4}+254.6 s^{3}} \\
& +\frac{+1.650 \times 10^{5} s^{2}+2.643 \times 10^{5} s+1.378 \times 10^{5}}{+1103 s^{2}+1823 s+172.4}
\end{aligned}
$$

Furthermore, substituting the result of Diophantine equation (4) into eqs. (6), (7), we arrive at

$$
\begin{aligned}
& T_{1}(s)=\frac{3 s+9}{s^{2}+8 s+7} \\
& T_{2}(s)=\frac{s^{4}+16 s^{3}+86 s^{2}+176 s+105}{s^{4}+15 s^{3}+69 s^{2}+97 s+42}
\end{aligned}
$$

Fig. 2 (a) shows the output response of the proposed model matching control for continuous-time system with step disturbance $(w(t)=0.5)$, when $\rho=0.01$. Fig. 2 shows that the proposed method is applicable to continuous-time system, and the plant output

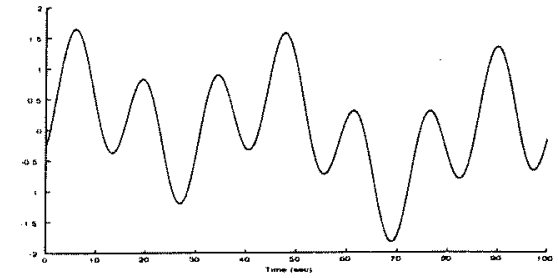

(a) $--; y(t)$ (plant output); - $y_{m}(t)$ (reference output)

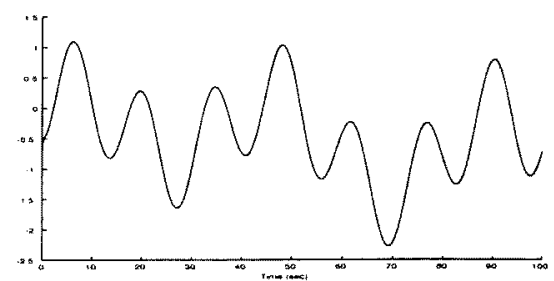

(b) $u(t)$ (plant input)

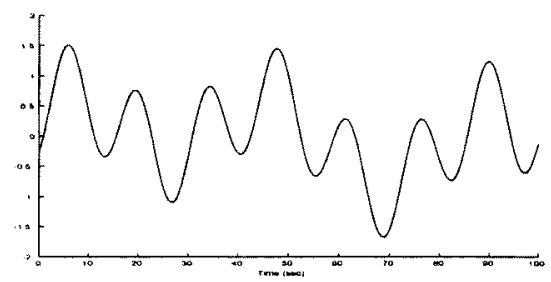

(c) $r(t)$ (reference input)

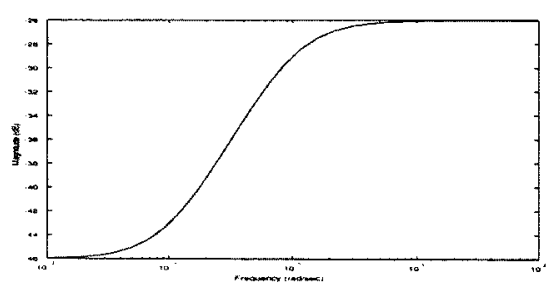

(d) $S S(s)$ (sensitivity function)

Fig. 3. Result of model matching control with step disturbance $(w(t)=0.5, \rho=0.1)$

can converge to the desired output $y_{m}(t)\left(y_{m}(t)=\right.$ $\left.G_{m}(s) r(t)\right)$ quickly in the presence of disturbance. Furthermore, when $\rho=0.1$, the model matching control for continuous-time system with step disturbance $(w(t)=0.5)$ is shown in Fig. 3. From Fig. 2 and Fig. 3, it is seen that when we choose small $\rho$, the effect of the disturbance can be decoupled from the plant output.

Example 2: Let us consider the case when the polynomial $A(s)$ has unstable poles, and the plant with external disturbance described by the following equation

$$
y(t)=\frac{B(s)}{A(s)} r(t)+w(t)=\frac{s^{2}+6 s+5}{s^{2}+s-6} r(t)+w(t)
$$

where $A(s)$ has a stable pole at -3 and an unstable pole at 2 , and $B(s)$ has two stable zeros at -1 and -5 .

Furthermore, Fig. 4 shows the external disturbance.

The reference model $G_{m}(s)$ is given by 


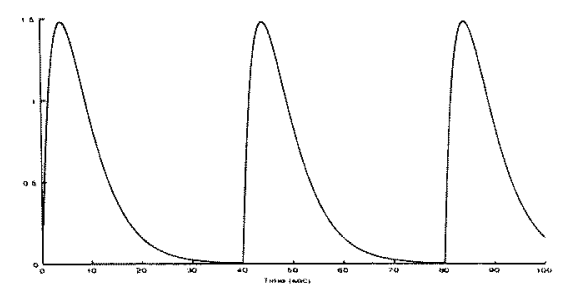

Fig. 4. External disturbance

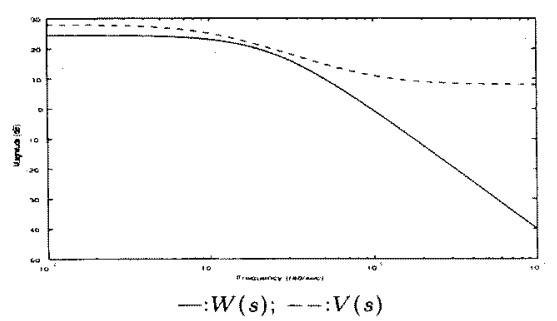

Fig. 5. $W(s)$ and $V(s)$

$$
G_{m}(s)=\frac{s^{2}+7 s+12}{s^{2}+10 s+16}
$$

Thus, we have $A^{+}(s)=s+3, A^{-}(s)=s-2$.

Choose the small constant $\rho=0.01$, and stable polynomials $T_{0}(s)=s^{2}+4 s+3, T_{3}(s)=s^{3}+6 s^{2}+11 s+6$, $V(s)=2.513(s+1) /(s+0.1005)$.

The norms of $W(s)$ and $V(s)$ are shown in Fig. 5.

From eq. (23), we obtain

$$
L(s)=\frac{-0.746 s-4.714}{s^{2}+10.29 s+25.14}
$$

Solve Diophantine equation (16), and from eqs. (17), (18), $N(s) / M(s)$ is

$$
\frac{N(s)}{M(s)}=\frac{-252.3 s^{3}-2344 s^{2}-5259 s-3161}{s^{3}+7.101 s^{2}+12.7 s+1.206}
$$

Furthermore, substituting the result obtained that solve Diophantine equation (4) into eqs (6), (7), we arrive at

$$
\begin{aligned}
& T_{1}(s)=\frac{5.714 s+17.14}{s^{2}+7.286 s+6.286} \\
& T_{2}(s)=\frac{s^{4}+11 s^{3}+43 s^{2}+69 s+36}{s^{4}+13.29 s^{3}+55 s^{2}+74.14 s+31.43}
\end{aligned}
$$

Fig. 6 (a) shows the output response of the proposed model matching control for continuous-time system with periodic disturbance, when $\rho=0.01$. Fig. 6 shows that the proposed method is applicable to continuous-time system, and the plant output can converge to the desired output $y_{m}(t)$ quickly in the presence of disturbance. Furthermore, when $\rho=0.1$, the model matching control for continuous-time system with periodic disturbance is shown in Fig. 7. From Fig. 6 and Fig. 7 , it is seen that when we choose small $\rho$, the effect of the disturbance can be decoupled from the plant output.

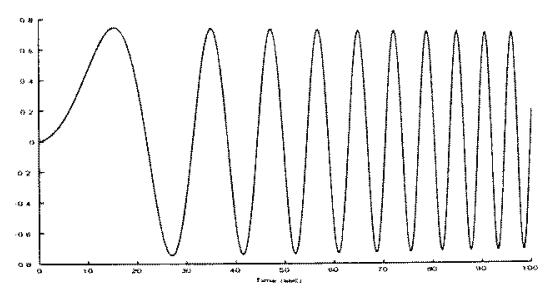

(a) $--: y(t)$ (plant output); - $y_{m}(t)$ (reference output)

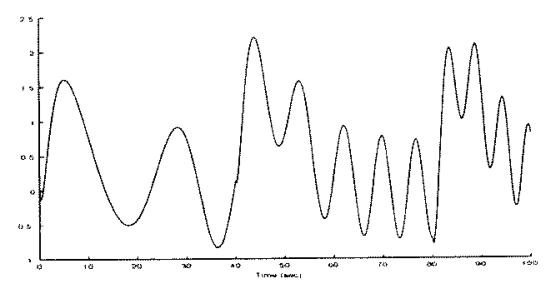

(b) $u(t)$ (plant input)

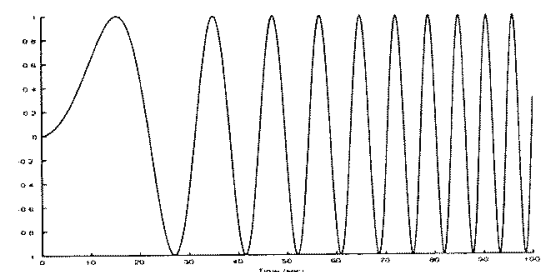

(c) $r(t)$ (reference input)

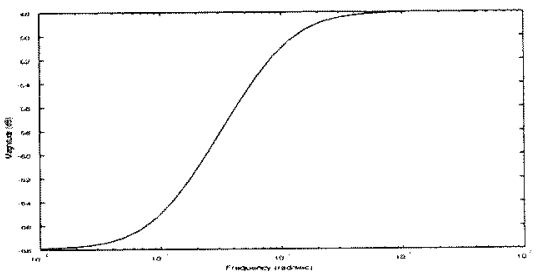

(d) $S S(s)$ (sensitivity function)

Fig. 6. Result of model matching control with disturbance $(\rho=0.01)$

Example 3: Let us consider the case when the plant with external disturbance described by the following equation

$$
y(t)=\frac{B(s)}{A(s)} r(t)+w(t)=\frac{s+1}{s^{2}+s-6} r(t)+w(t)
$$

where $A(s)$ has a stable pole at -3 and an unstable pole at 2 , and $B(s)$ has a stable zeros at -1 .

The reference model $G_{m}(s)$ is given by

$$
G_{m}(s)=\frac{s+3}{s^{2}+10 s+16}
$$

Furthermore, Fig. 8 shows the external disturbance.

Choose the small constant $\rho=0.01$, and stable polynomials $T_{0}(s)=s^{2}+3.6 s+3.15, T_{3}(s)=s^{3}+180 s^{2}+$ $10724 s+211200, V(s)=1.508 /(s+0.1005)$.

The norms of $W(s)$ and $V(s)$ are shown in Fig. 9.

From eq. (27), we obtain

$$
L(s)=\frac{1.508 \times 10^{8} s-5.447 \times 10^{12}}{s^{2}+1.508 \times 10^{8} s+4.524 \times 10^{8}}
$$




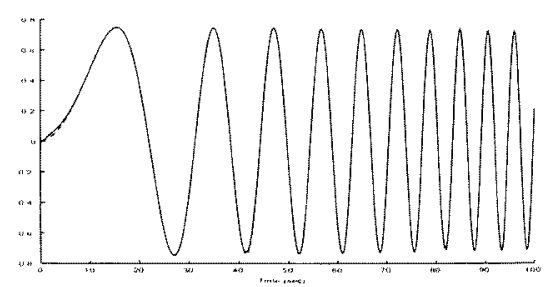

(a) $--: y(t)$ (plant output); -:ym $(t)$ (reference output)

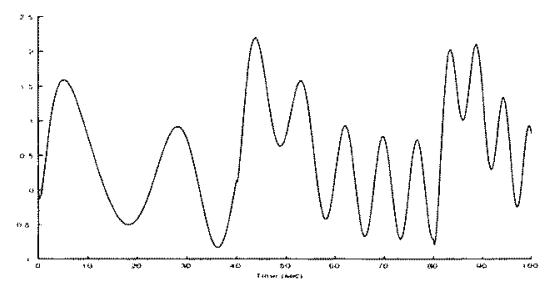

(b) $u(t)$ (plant input)

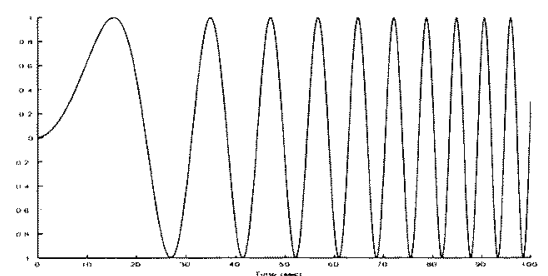

(c) $r(t)$ (reference input)

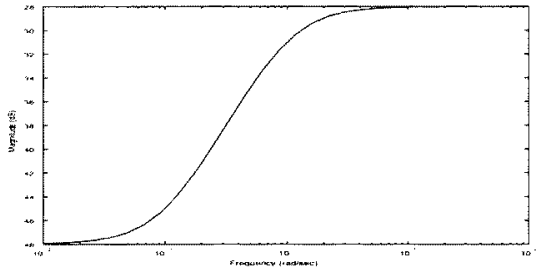

(d) $S S(s)$ (sensitivity function)

Fig. 7. Result of model matching control with disturbance $(\rho=0.1)$

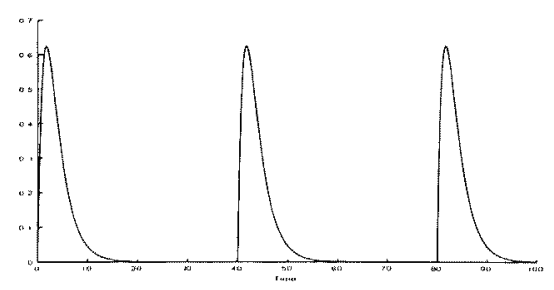

Fig. 8. External disturbance

Solve Diophantine equation (16), and from eqs. (17), (18), $N(s) / M(s)$ is

$$
\begin{aligned}
\frac{N(s)}{M(s)}= & \frac{1.508 \times 10^{8} s^{3}+2.714 \times 10^{10} s^{2}}{s^{3}+3995 s^{2}} \\
& * \frac{+1.617 \times 10^{12} s+3.185 \times 10^{13}}{+6.353 \times 10^{5} s+3.061 \times 10^{8}}
\end{aligned}
$$

Furthermore, substituting the result obtained that solve Diophantine equation (4) into eqs (6), (7), we arrive at

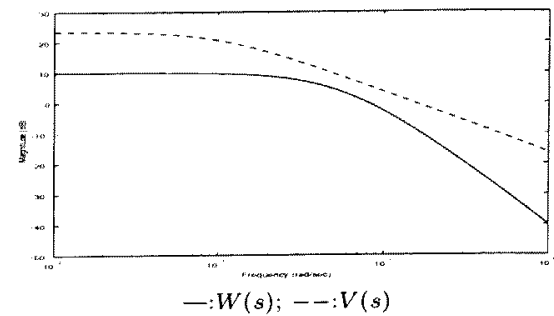

Fig. 9. $W(s)$ and $V(s)$

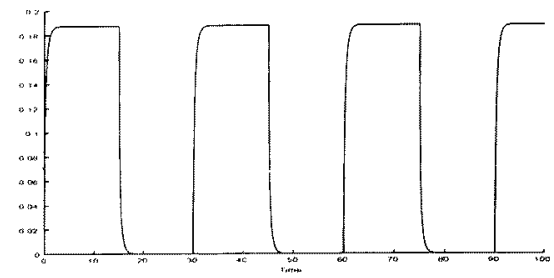

(a) $--: y(t)$ (plant output); -: $y_{m}(t)$ (reference output)

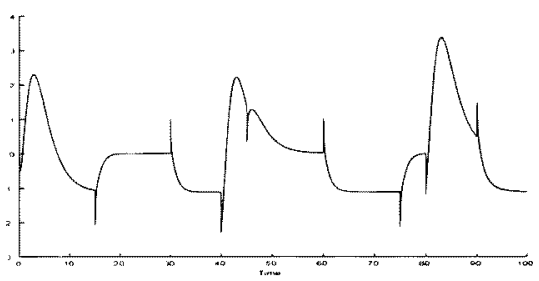

(b) $u(t)$ (plant input)

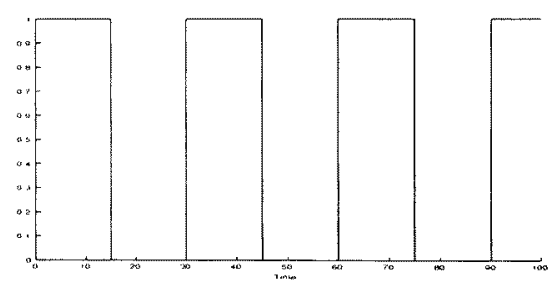

(c) $r(t)$ (reference input)

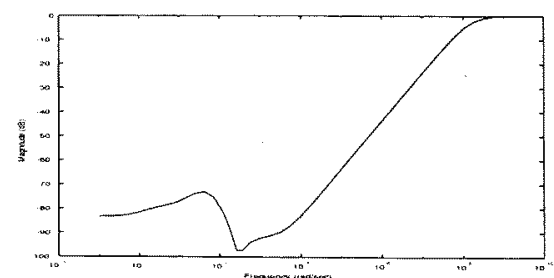

(d) $S S(s)$ (sensitivity function)

Fig. 10. Result of model matching control with disturbance $(\gamma=0.01)$

$$
\begin{aligned}
& T_{1}(s)=\frac{37.59 s+116.1}{s^{2}+12.6 s+10.96} \\
& T_{2}(s)=\frac{s^{3}+6.6 s^{2}+13.95 s+9.45}{s^{3}+13.6 s^{2}+23.56 s+10.96}
\end{aligned}
$$

Fig. 10 shows the output response of the proposed model matching control for continuous-time system with the periodic disturbance, when $\rho=0.01$. Fig. 10 shows that the proposed method is applicable to continuous-time system, and the plant output can 


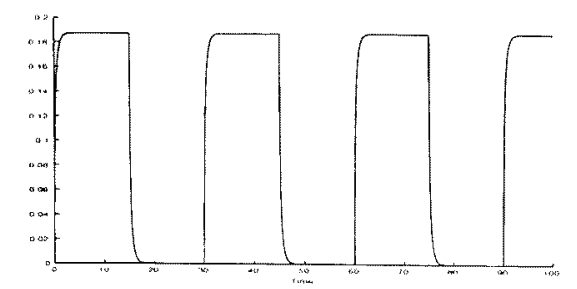

(a) $--: y(t)$ (plant output); - $-y_{m}(t)$ (reference output)

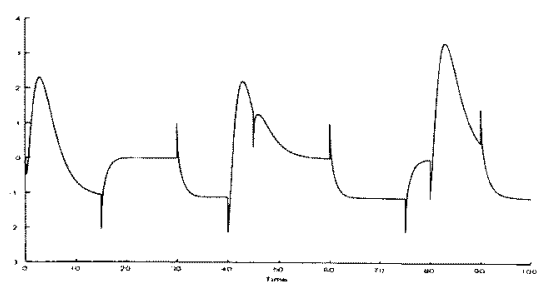

(b) $u(t)$ (plant input)

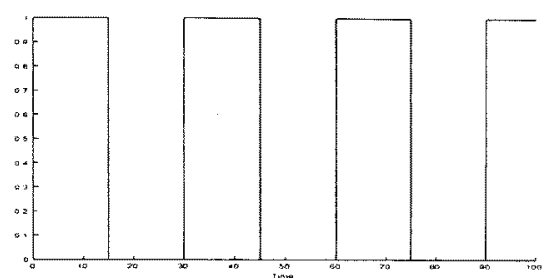

(c) $r(t)$ (reference input)

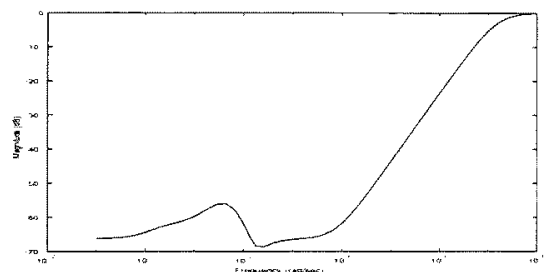

(d) $S S(s)$ (sensitivity function)

Fig. 11. Result of model matching control with disturbance $(\gamma=0.1)$

converge to the desired output $y_{m}(t)$ in the presence of periodic disturbance. Furthermore, when $\rho=0.1$, the model matching control for continuous-time system with the periodic disturbance is shown in Fig. 11. From Fig. 10 and Fig. 11, it is seen that when we choose small $\rho$, the effect of the disturbance can be decoupled from the plant output.

Furthermore, it can also be seen from simulation results that when we use the input $u(t)$ in eq. 5 , we can assure that the system input and output remain bounded at any time for continuous-time system with arbitrarily bounded disturbance.

\section{Conclusion}

We have proposed a new technique to design a controller with two degree of freedom such that the stability of the system can be assured in presence of disturbances. Furthermore, the transfer function of the system from the reference input $r(t)$ to plant output $y(t)$ can be made to match some reference model transfer

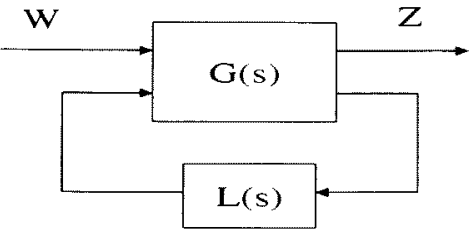

Fig. 12. $H_{\infty}$ control system

function. The system input and output remain bounded at any time for continuous-time system with arbitrarily bounded disturbance.

Appendix: The case of $n>m$ and $\nu-\mu \geq n-m$ Let us define the following equation

$$
\bar{A}(s)=\frac{\bar{M}(s) R(s) A(s) V(s)}{\gamma T_{0}(s) T_{3}(s)}=\frac{\bar{B}_{1}(s)}{\bar{A}_{1}(s)} \ldots \ldots
$$

where $V(s)=V_{n}(s) / V_{d}(s), \operatorname{deg} V_{n}(s)=l_{1}, \operatorname{deg} V_{d}(s)=$ $l_{2}, \operatorname{deg} \bar{A}_{1}(s)=l+\xi+l_{2}, \operatorname{deg} \bar{B}_{1}(s)=l+l_{1}+\xi$. Furthermore, $\bar{B}(s)$ is defined as

$$
\bar{B}(s)=\frac{B_{m}(s) R(s) A(s) V(s)}{\gamma T_{0}(s) T_{3}(s)}=\frac{\bar{B}_{2}(s)}{\bar{A}_{1}(s)}
$$

where $\operatorname{deg} \bar{B}_{2}(s)=\mu+\nu+l+l_{1}$.

From eqs. (25) and (26), it is possible to write

$$
\begin{aligned}
& \bar{A}(s)=C_{1}^{T}\left(s I-A_{1}\right)^{-1} B_{1} \\
& \bar{B}(s)=C_{2}^{T}\left(s I-A_{1}\right)^{-1} B_{2}
\end{aligned}
$$

where, $A_{1}$ is a $\left(l+\xi+l_{2}\right) \times\left(l+\xi+l_{2}\right)$ matrix, $B_{1}, C_{1}$, $B_{2}$ and $C_{2}$ are $\left(l+\xi+l_{2}\right) \times 1$ vectors. Furthermore, using eqs. (20), (25), (26), we obtain

$$
|\bar{A}(s)-\bar{B}(s) L(s)|<1, \quad s=j \omega, \forall \omega \ldots \ldots
$$

Equation (29) can be rewritten as

$$
\begin{aligned}
& \bar{A}(s)-\bar{B}(s) L(s) \\
& \quad=\bar{A}(s)-\bar{B}(s) L(s)(I-0 \cdot L(s))^{-1} I .
\end{aligned}
$$

Let the state space controller be $L(s)$, then the block diagram of the system can be given as in Fig. 12 .

$$
G(s)=\left[\begin{array}{cc}
\bar{A}(s) & -\bar{B}(s) \\
I & 0
\end{array}\right]
$$

Using eqs. (27), (28), and (31), we obtain

$$
G(s)=\left[\begin{array}{cc|cc}
A_{1} & 0 & B_{1} & 0 \\
0 & A_{1} & 0 & B_{2} \\
\hline C_{1} & -C_{2} & 0 & 0 \\
0 & 0 & I & 0
\end{array}\right]
$$

The state feedback $H_{\infty}$ controller $L(s)$ can be obtained by solving the $H_{\infty}$ problem given in Fig. 12(5),(19),(20).

(Manuscript received March 31, 1999, revised April $10,2000)$ 


\section{References}

(1) V. Kučera, Analysis and Design of Discrete Linear Control Systems, Prentice Hall, pp. 335, 1991.

(2) P. N. Paraskevopoulos: "Exact transfer function design using output feedback", IEE Proceeding-D, vol. 123, no. 8, pp. 831-834, 1976.

(3) V. Kučera: "Exact model matching, polynomial equation approach", Int. J. Syst. vol. 12, pp. 1477-1484, 1981.

(4) A. I. G. Vardulakis: "proper rational matrix Diophantine equations and the exact model matching problem", IEEE Trans. Automat. Contr., vol. AC-29, pp. 475-477, Oct. 1984

(5) B. A. Francis: A Course on $H_{\infty}$ Control Theory, Lecture Notes in Control and Information Sciences, Springer-Verlag, 1987

(6) B. C. Chang and J. B. Pearson: "Optimal disturbance reduction in linear multivariable systems", IEEE Trans. Automat. Contr., vol. AC-29, no. 10, pp. 880-887, 1984.

(7) M. Vidyasagar: Nonlinear Systems Analysis, Prentice Hall, pp. 337-340, 1993.

(8) K. Glover: "All optimal Hankel-norm approximation of linear multivariable systems and their $L_{\infty}$ error bound", Int. J. Contr., vol. 39, pp. 1115-1193, 1984.

(9) J. C. Doyle: "Lecture notes in advances in multivariable control", ONR/ Honeywell Workshop, Minneapolis, MN, 1984.

(10) J. A. Ball and C. M. Ran: "Optimal Hankel-norm model reductions and Wiener-Hopf factorizations I: The canonical case", SIAM J. Contr. Optimize., vol. 25, no. 2, pp.. 362-382, 1987.

(11) PH. Delsatre, Y. Genin and Y. Kamp: "On the role of the Nevanlina-Pick problem in circuit and system theory", Int. J. Circuit Theory Application, vol. 9, pp. 177-187, 1981

(12) P. Gahint and P. Apkarian: "An LMI-based parameterization of all $H_{\infty}$ controller with application", Proc. of the 32nd Conference on Decision and control, San Antonio, Texas, pp. 656-661, 1993

(13) V. Kučera, Discrete Linear Control: The Polynomial Equation Approach, John Wiley \& Sons, Inc., 1979.

(14) H. Kwakernaak: "The polynomial approach to $H_{\infty}$ optimal regulation", In $H_{\infty}$ - Control Theory, Lecture notes in Mathematics, vol. 1496, Springer-Verlag, pp. 141-221, 1991

(15) T. E. Dajaferis, Robust Control Design: A polynomial approach, Kluwer Academic Publishers, 1995.

(16) S. P. Bhattacharyya, H. Chapellart and L. H. Keel, Robust Control: The Parametric Approach, Prentice Hall, 1995.

(17) J. Lu and T. Yahagi: "New design method for model reference adaptive control for nonminimum phase discrete-time systems with disturbances", IEE Proceeding-D, vol. 140, no. 1, pp. 34-40, 1993.

(18) J. Lu and Takashi Yahagi: "Discrete-time multivariable model reference adaptive control with disturbances", Trans. IEE of Japan, vol. 118-C, no. 9, pp. 1362-1368, 1998.

(19) K. Zhou, J. C. Doyle, and K. Glover: Robust Optimal Control, Prentice Hall, 1996

(20) J. Lu, T. Yahagi: "A design method of robust model matching control for continuou-time systems in the presence of unmodeled dynamics", Trans. IEE of Japan, vol. 118-C, no. 5, pp. 737-742, 1998.

Jianming Lu (Member) received the M.S., and

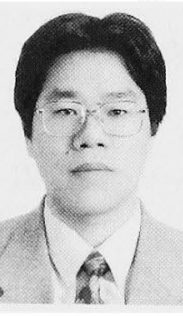
$\mathrm{Ph} . \mathrm{D}$. degrees from Chiba University, Japan, in 1990 and 1993, respectively. In 1993, he joined Chiba University, Chiba, Japan, as an Assistant Professor in the Department of Information and Computer Sciences. Since 1994 he has been with the Graduate School of Science and Technology, Chiba University, and in 1998 he was promoted to
Associate Professor in the Graduate School of Science and Technology, Chiba University. His current research interests are in the theory and applications of digital signal processing and control theory. Dr. Lu is a member of IEICE(Japan), SICE(Japan), IEEJ(Japan) and JSME(Japan).

Kyohei Ishihata (Non-member) received the B.E.,

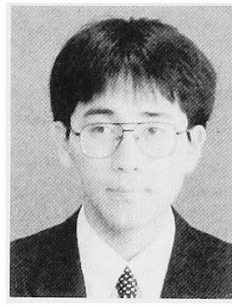
and M.S. degrees from Chiba University, Japan, in 1998 and 2000, respectively. Currently he is completing the $\mathrm{Ph}$.D.degree. His current research interests are in model matching control and computer control theory.

Takashi Yahagi (Non-member) received the B.S.,

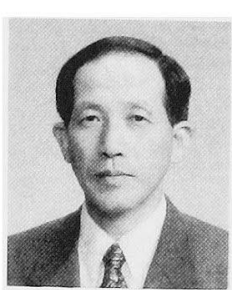
M.S., and Ph.D. degrees all in electronics engineering from the Tokyo Institute of Technology, Tokyo, Japan, in 1966, 1968 and 1971, respectively. In 1971, he joined Chiba University, Chiba, Japan, as a Lecturer in the Department of Electronics Engineering. From 1974 to 1984 he was an Associate Professor, and in 1984 he was promoted to Professor in the Department of Electrical Engineering. From 1989 to 1998, he was with the Department of Information and Computer Sciences. Since 1998 he has been with the Graduate School of Science and Technology, Chiba University. His current research interests are in the theory and applications of digital signal processing and other related areas. He is the author of "Theory of Digital Signal Processing", Vols.1-3(1985, 1985, 1986), and "Digital Signal Processing and Basic Theory" (1996), and the co-author of "Digital Signal Processing of Speech and Images" (1996), "VLSI and Digital Signal Processing" (1997), "Multimedia and Digital Signal Processing" (1997), "Neural Network and Fuzzy Signal Processing" (1998), "Communications and Digital Signal Proceming" (1999), "Fast Algorithms and Parallel Signal Processing "(2000) (Corona Pub. Co., Ltd., Tokyo, Japan). He is the editor of the "Library of Digital Signal Processing" (Corona Pub. Co., Ltd., Tokyo, Japan). Since 1999 he has been Chairman of the IEEE Japan Chapter of Signal Processing Society. Dr. Yahagi is a member of IEEE (USA), IEICE (Japan), The New York Academy of Sciences (USA), ISCIE(Japan). 